\title{
Paysages du maïs au Mexique
}

Comment la diversité est-elle structurée et que voulons-nous conserver?

Maize landscapes in Mexico: how is diversity structured and what do we want to conserve?

Paisajes del maíz en México: ¿cómo está estructurada la diversidad y qué queremos conservar?

\section{Hugo Perales}

\section{OpenEdition \\ Journals}

Édition électronique

URL : https://journals.openedition.org/ethnoecologie/7520

DOI : $10.4000 /$ ethnoecologie. 7520

ISSN : 2267-2419

Éditeur

Laboratoire Éco-anthropologie

\section{Référence électronique}

Hugo Perales, « Paysages du maïs au Mexique », Revue d'ethnoécologie [En ligne], Supplément 2 | 2021 mis en ligne le 25 novembre 2021, consulté le 20 décembre 2021. URL : http://

journals.openedition.org/ethnoecologie/7520 ; DOI : https://doi.org/10.4000/ethnoecologie.7520

Ce document a été généré automatiquement le 20 décembre 2021.

\section{(c) (i) $(\Theta$}

Revue d'ethnoécologie est mis à disposition selon les termes de la licence Creative Commons Attribution - Pas d'Utilisation Commerciale - Pas de Modification 4.0 International. 


\section{Paysages du maïs au Mexique}

Comment la diversité est-elle structurée et que voulons-nous conserver?

Maize landscapes in Mexico: how is diversity structured and what do we want to conserve?

Paisajes del maíz en México: ¿cómo está estructurada la diversidad y qué

queremos conservar?

Hugo Perales

\section{NOTE DE L'AUTEUR}

Une version similaire de ce document a été présentée à la réunion « Regards croisés sur la gestion de la biodiversité cultivée : acteurs, interprétations et stratégies d'action, le cas du mais ", le 11 septembre 2014, au Muséum national d'Histoire naturelle, Paris et à la reunion « Utilization and conservation of crop wild relative and landrace diversitet for crop improvement », organisée par PGR Secure Consortium et EUCARDIA, Cambridge, UK, Junio 16-20, 2014 ; et chacune publiée dans Maxted et al. (2016).

\section{Introduction}

1 Jusqu'au début du $\mathrm{xx}^{\mathrm{e}}$ siècle les agriculteurs ont été pratiquement les seuls pourvoyeurs de semences pour l'agriculture. Cent ans après, l'amélioration génétique standardisée est parvenue à s'imposer comme source des semences utilisées dans les cultures commerciales dans le monde. Concernant la décennie de 1960, plus de $95 \%$ du maïs semé aux États-Unis l'était déjà avec des semences commerciales (Fernandez C. 2004); d'autres cultures comme le blé, le riz, et le soja se trouvent dans une situation similaire dans plusieurs pays du monde.

Cependant, au Mexique, on calcule que seulement de $18 \%$ à $25 \%$ des semences de maïs proviennent directement d'industries ou d'institutions publiques (Aquino et al. 2001, Luna et al. 2012). Ce n'est pas là un phénomène mineur, chaque année quelques 2,5 
millions d'agriculteurs sèment environ 8 millions d'hectares (Eakin et al. 2014). Cela implique que plus de 2 millions d'agriculteurs produisent eux-mêmes leur semence et la sèment sur plus de 4 millions d'hectares, et ceux-ci produisent plus de 8 millions de tonnes de maïs, qui suffisent à nourrir plus de 25 millions de Mexicains.

L'amélioration génétique menée par des professionnels a eu tant de succès que nous sommes arrivés à croire que les agriculteurs n'ont pas leur place dans l'amélioration et l'évolution future de nos cultures. Ces dernières années cela s'est intensifié avec les progrès de la biotechnologie et les cultures transgéniques. En 2014, les variétés transgéniques sont semées sur plus de 180 millions d'hectares dans le monde (Marshall 2015). Cependant, il est indispensable de se demander si l'importance de l'amélioration génétique institutionnelle peut à l'avenir occuper tout l'espace en remplaçant toutes les variétés traditionnelles; et, dans le cas contraire, quelles en seraient les conséquences pour la culture du maïs au Mexique.

Dans cet article on présentera un panorama des différents facteurs qui structurent la diversité du maïs natif au Mexique, on proposera une interprétation qui prendra en compte l'amélioration évolutive et la sécurité alimentaire et on mettra en avant que ce qu'il faut conserver ce ne sont pas les gènes pour les sélectionneurs mais bien plutôt la présence des agriculteurs comme agents effectifs dans le processus évolutif du maïs.

\section{Antécédents}

5 La conservation des ressources génétiques des plantes a été envisagée en vue de trouver les moyens de perpétuer les gènes existants pour que les sélectionneurs en disposent et puissent les utiliser dans le développement de variétés produites par des institutions. De même que pour les espèces sauvages, nous pouvons faire de la conservation des ressources génétiques des plantes cultivées selon deux grandes modalités que nous caractérisons comme étant in situ (là où elles se trouvent) et ex situ (en dehors). Pour les espèces sauvages aussi bien que cultivées, la conservation in situ signifie que les plantes ou animaux qu'il nous importe de conserver sont maintenus dans leur lieu d'origine, ou vivent par leurs propres moyens. Dans le cas de 1 'agriculture cela veut dire dans les champs des agriculteurs qui les cultivent. Dans la conservation ex situ les espèces qui nous intéressent sont rassemblées et on en prend soin selon des conditions contrôlées. C'est ce que nous faisons dans des banques de gènes, où nous gardons les graines dans des réfrigérateurs ou des congélateurs, nous cultivons les plantes dans des jardins botaniques, nous maintenons les cellules dans des cultures de tissus ou sous d'autres formes qui nous permettent de conserver les variétés ou gènes. Dans le cas des espèces sauvages il y a une préférence évidente pour la conservation in situ, contrairement à la préférence pour la conservation ex situ en ce qui concerne les espèces cultivées.

6 Cette différence dans le choix de la méthode de conservation pour les espèces sauvages et pour les espèces cultivées n'est pas le fruit du hasard. Quand on a institutionnalisé le mode de conservation des ressources génétiques agricoles, on a considéré qu'on ne pouvait pas dépendre des agriculteurs pour assurer et mettre à disposition la diversité génétique pour l'avenir. Otto H. Frankel (1970) a conclu, il y a presque 50 ans, que « les procédés de conservation sur le long terme des populations 'primitives' dans leur habitat naturel étaient beaucoup plus incertains que pour les espèces sauvages ». C'està-dire qu'on s'attendait à ce que les variétés commerciales soient toujours supérieures 
et à ce qu'elles remplacent les variétés traditionnelles; si les agriculteurs ne changeaient pas les variétés traditionnelles par les commerciales, ils perdraient en production et en argent. (Plucknett et al. 1987, Frankel et al. 1995). On n'aurait donc pas intérêt à dépendre d'eux.

7 Le regard porté sur l'importance de la conservation des ressources génétiques agricoles à la ferme a changé depuis les années 90 . D'abord, on a commencé à se documenter sur les cas de conservation des variétés traditionnelles malgré la présence de variétés commerciales (Brush 1995). Comme on va le décrire, les raisons pour maintenir les variétés traditionnelles sont multiples, mais le fait est que les variétés commerciales $\mathrm{n}$ 'ont pas remplacé les variétés traditionnelles dans de nombreux endroits. De plus, la Convention pour la Diversité Biologique (Naciones Unidas 1993) a mis en avant la nécessité de la conservation in situ également pour les plantes cultivées et a suscité un climat politique favorable à la vision actuelle de complémentarité des deux sortes de conservation des ressources génétiques agricoles. Cependant, la préférence pour la conservation ex situ et son influence sur la conservation à la ferme pèsent toujours dans la conservation de la biodiversité cultivée.

8 La principale cause responsable de cet état de choses est le fait que l'on continue de concevoir les gènes en fonction des généticiens et des sélectionneurs. Parmi les avantages de la conservation in situ, on inclut toute la complexité des variations sur champ, qui reste un laboratoire naturel, dans lequel les processus évolutifs continuent de créer de nouveaux gènes et où sont conservées aussi des espèces associées. Enfin on a souligné que la connaissance locale, en plus de permettre d'avoir de nouveaux échantillons, peut constituer un mécanisme d'adaptation face au changement climatique. Cependant, bien que l'on reconnaisse qu'il est nécessaire de maintenir et $d$ 'augmenter les bénéfices privés des agriculteurs pour renforcer la conservation in situ (Brush 2000, Bellon 2004), on envisage la conservation in situ comme un complément à la conservation ex situ. La conservation in situ est vue comme un simple transfert de la conservation ex situ, avec essentiellement des objectifs similaires. Selon cette conception maintenir les variétés traditionnelles ou les systèmes agricoles traditionnels "sans changement ", ou conserver quelques populations de chaque sorte (comme échantillons dits « de garde»), parmi les milliers ou centaines de milliers qui sont semés, paraît sensé et c'est une stratégie courante. Cette vision de la conservation in situ de l'agrobiodiversité, un peu statique et conçue en vue de produire des gènes, comme une extension des banques de gènes, est si courante que de De Boef et al. (2012) considèrent qu'il s'agit d'un oxymore et préfèrent parler de conservation dans les champs des agriculteurs (ou on-farm conservation, expression préférée par d'autres auteurs pour faire la distinction avec les espèces sauvages, voir Maxted et al. 2002). Autrement dit, le processus est de nature si dynamique qu'ils jugent que ce n'est pas à proprement parler de la "conservation", au sens d'empêcher les changements et maintenir des ressources génétiques dans un état originel particulier. Cette opinion part d'un concept particulier de la conservation qui s'applique mal en agriculture. Il y a 50 ans Bennett a signalé que « le but de la conservation n'est pas de capter le moment présent du temps évolutif, ceci serait dépourvu de vertu particulière, mais de conserver le matériel pour qu'il continue à évoluer ». (Frankel $1970: 476$ ). D'autre part, aujourd 'hui encore, les banques de gènes sont peu utilisées par les sélectionneurs et il est difficile d'imaginer que les variétés traditionnelles conservées in situ, dont la caractérisation est plus coûteuse et plus limitée, seront très utilisées par les sélectionneurs dans des conditions normales. Mais la conservation in situ a d'abord 
pour but le bien-être des agriculteurs et de leur familles (Brush 2000, Bellon 2004, Jarvis et al. 2011, De Boef et al. 2012) et nous demandons que soit reconnu ce fait que les gènes ne sont qu'un sous-produit de la génération de variétés obtenues par les agriculteurs et ne représentent donc qu'un objectif secondaire. Si les variétés cessent d'avoir un intérêt pour les agriculteurs, la conservation in situ agricole deviendra un phénomène superflu, soutenu artificiellement par des subventions, et ayant peu de différence avec la conservation ex situ. C'est parce qu'elles répondent aux besoins des agriculteurs, à leur sécurité alimentaire et à leurs marchés, que ces variétés sont conservées car, au moins pour le maïs au Mexique, dans la plupart des endroits où dominent les variétés traditionnelles il n'y a pas d'alternatives qui leur soient supérieures. La conservation in situ demande à être considérée du point de vue des agriculteurs et non des généticiens. $C^{\prime}$ est-à-dire qu'il faut la considérer en vue de répondre aux objectifs des paysans et ensuite seulement, pour satisfaire des aspects de la société en général (par exemple, les gènes pour les généticiens).

\section{Production du maïs au Mexique}

Le climat économique pour la production du maïs au Mexique a changé ces 20 dernières années (Appendini 2014), mais, la conservation du maïs dans les champs des agriculteurs n'a pas été fortement affectée. En 1994 est entré en vigueur l'accord de libre-échange nord-américain (ALENA) signé entre les États-Unis, le Canada et le Mexique. L'ALENA inclut des mesures pour maintenir les tarifs pour le maïs car on a considéré que la production locale ne pouvait pas concurrencer celle des États-Unis et du Canada (Nadal 1999). On s'attendait à ce que la production de maïs décline au Mexique avec l'érosion de sa biodiversité (Nadal 2000). Plus de 20 ans après l'entrée en vigueur de l'ALENA on a enregistré des changements dans la production régionale (Sweeneet et al. 2013), le plus remarquable étant l'augmentation de la production et 1 'importance de Sinaloa. Il y a 25 ans, à Sinaloa, on produisait environ 0,3 million de tonnes (Mt), en 2014 on a produit plus de $5 \mathrm{Mt}$, ce qui représente environ le quart de tout le maïs produit au Mexique (Eakin et al. 2014, SIAP 2016). Cette restructuration du marché du maïs au Mexique a été promue par des politiques de soutien aux grands producteurs privés, et en diminuant en partie le soutien apporté aux producteurs assurant leur subsistance (Appendini 2014, Fox \& Haight 2010). Cependant, au niveau national, les agriculteurs n'ont pas abandonné le maïs (Eakin et al. 2014). La surface totale semée au Mexique est restée relativement stable entre 1994 et 2014, autour de 8 millions d'hectares et la production totale est passée de 18,1 à 22,7 millions de tonnes dans la même période (1993-2013). Le nombre d'agriculteurs semant du maïs est resté stable dans les deux recensements disponibles, 2,75 millions en 1991 et 2,83 millions en 2007. Ceci montre que les prédictions d'une dégradation de la production de maïs étaient fausses.

10 La production de maïs au Mexique résulte d'une structure double «moderne/ paysanne » qui existe encore maintenant (Hewitt 1976, Eakin et al. 2014). La production "moderne » se trouve majoritairement dans le nord du pays et est associée à la disponibilité de l'irrigation ( $18 \%$ de la superficie totale de maïs); dans ce type de production, on utilise de grandes quantités d'intrants et de semences commerciales. Les rendements dans ces systèmes peuvent être aussi importants que ceux de $n$ 'importe quelle autre région du monde et représentent $43 \%$ de toute la production. 
Mais au Mexique la majeure partie du maïs est semée dans des terres sans irrigation et donc dépendantes du régime pluviométrique ( $82 \%$ du total) et près de $85 \%$ des agriculteurs sèment chacun moins de cinq hectares de maïs.

\section{Semis des variétés traditionnelles et commerciales}

11 Nous ne disposons pas d'estimations fiables de la quantité de semences commerciales de maïs semées au Mexique. Aquino et al. (2001) ont calculé que les variétés commerciales représentaient $22 \%$ du total de semences requises en 1999 ( $15 \%$ de semences hybrides et $7 \%$ de variétés à pollinisation ouverte). Luna et al. (2012) ont signalé que la quantité était de $19 \%$ en 2009 et García et Ramírez (2014) l'ont estimée de $42 \%$ (une surestimation est très possible car les données originaires de l'usage de variétés commerciales ne sont pas clairement définies). C'est-à-dire que près de $80 \%$ de la semence au Mexique provient des récoltes des agriculteurs. Il ne s'agit pas uniquement de variétés traditionnelles (Bellon \& Risopoulos 2001, Bellon et al. 2006, Aguirre et al. 2000), il y a aussi des variétés commerciales recyclées, (c'est-à-dire qui résultent de générations avancées de variétés commerciales) ou créolisées (c'est-à-dire de générations avancées de variétés commerciales avec l'introgression de pollen issu des variétés traditionnelles). Nous ne disposons pas d'une estimation fiable au niveau national de la quantité de semence recyclée ou créolisée ; les estimations locales vont de $10,8 \%$ de variétés recyclées et $27 \%$ de créolisées dans des communautés de la Frailesca, Chiapas (Bellon et al. 2006) à $4,4 \%$ et $11 \%$, respectivement, dans des communautés de la Côte d'Oaxaca (Bellon et al. 2006) ou seulement de 3,5\% de créolisées à Guanajuato (Aguirre et al. 2000). On peut donc assurer que lorsque moins de $50 \%$ de la semence au Mexique est représentée par des variétés traditionnelles - bien que le chiffre puisse arriver à $75 \%$ - il n'y a pas d'indices que l'adoption et la distribution de variétés commerciales aient augmenté dans les dernières décennies. En 2009 le Gouvernement mexicain a mis en place un programme de soutien à la conservation de variétés de maïs natif dans les champs des agriculteurs (PROMAC, Programa de Maïs Criollo 2015). Le programme a consisté principalement en aides directes données à ceux qui sèment ces variétés près des Zones Naturelles Protégées ou à l'intérieur de celles-ci, et cela représente près de $0,4 \%$ des cultivateurs de maïs. On constate que la conservation du maïs dans les champs des agriculteurs est au Mexique un processus de facto robuste, indépendant de toutes les institutions, après plus de 60 ans d'efforts de sélection génétique.

\section{Diversité du maïs au Mexique}

$\mathrm{Au}$ Mexique nous classons la diversité en "espèces", selon la proposition faite à 1 'origine par Anderson \& Cutler (1942) et formalisée par Wellhausen et al. (1951). Anderson et Cutler (1942) ont défini le concept de race « de la façon la plus souple possible, comme un groupe d'individus présentant assez de caractéristiques communes pour permettre leur reconnaissance comme groupe" comme si c'était une classification naturelle susceptible de refléter des liens de phylogénèse. Sánchez \& Goodman (2000) ont comptabilisé 59 espèces pour le Mexique, bien que les échantillons de la banque de gènes soient très rares pour 12 de ces espèces et qu'il n'a pas été possible de dessiner leur distribution dans une étude récente (Perales \& Golicher 2014). 
Ces espèces ne sont pas uniformes, à l'intérieur de chacune d'elles il y a de grandes variations dans la couleur et la texture du grain, dans les caractères de la plante et dans sa phénologie. On trouve des épis avec des formes cylindriques et d'autres coniques, il y en a qui mesurent moins de huit centimètres et d'autres plus de $35 \mathrm{~cm}$. Les grains sont communément blancs ou jaunes, mais il y en a aussi qui sont de couleur crème, bleue, violine, rouge, orange, rose, café, violet et presque noire. On trouve aussi des grains marbrés, tachetés et avec d'autres types de coloration. Leur forme varie : arrondie, dentelée, plate, et même pointue ou striée et ils peuvent avoir une texture farineuse, cristalline ou cireuse; certains sont connus comme palomeros (des pigeons) ou éclatant sous l'effet de la chaleur. Il y a des grains qui sont allongés, d'autres courts et d'autres qui font deux centimètres de large; il y a des grains si petits qu'il en faut 70 pour avoir un gramme alors qu'il y en a d'autres qui pèsent plus d'un demi-gramme. Au Mexique, on sème le maïs depuis le niveau de la mer jusqu'à 2900 mètres, avec une température annuelle moyenne qui va de 26,6 Co à 11,3 Con et avec des précipitations annuelles qui varient de $200 \mathrm{~mm}$ à $4000 \mathrm{~mm}$. Ce fait caractérise la grande diversité physiologique du maïs qui, du point de vue agricole, est beaucoup plus importante que celle des formes et couleurs des épis et des grains observés. C'est sur la base de cette diversité physiologique que nous pouvons adapter des types de maïs à presque tous les environnements où nous en avons besoin.

Il est fréquent que dans les cas d'études dans les communautés ou dans les rapports des collectes pour des banques de gènes, on enregistre beaucoup d'espèces ou de variétés, mais le modèle courant c'est que peu d'entre elles dominent les environnements particuliers. Par exemple, même si pour l'État de Chiapas on rapporte la présence de 21 races dans les banques de gènes, 11 seulement représentent au moins $1 \%$ du total des collectes (Perales \& Hernández 2005). Dans un échantillon systématique pour le Chiapas pris entre 1999 et 2004 (119 communautés et 2073 foyers) on a trouvé huit races communes dont trois seulement représentaient plus de $80 \%$ de toutes les collectes (Brush \& Perales 2007). On a trouvé un résultat similaire à Oaxaca. Aragón et al. (2006) ont informé sur une large collecte $(\mathrm{n}=1818)$ et la présence de 35 races, mais un tiers de celles-ci comprenaient moins de dix exemplaires et huit races seulement représentaient $72 \%$ du total. On retrouve la même chose au niveau des communautés et des foyers, où il est fréquent qu'une à trois variétés représentent plus de $80 \%$ de la semence utilsée par une communauté et la moyenne des variétés semées par foyer tourne autour de 1,4 et dépasse rarement deux lots de semences (Dyer et al. 2014). Dyer et al. (2014) ont documenté une diminution du nombre de variétés par foyer entre 2002 et 2007 , mais on ne sait pas exactement comment cela affecte la diversité des espèces ou leurs variétés.

\section{Les facteurs qui structurent la diversité du maïs au Mexique}

14 L'altitude et l'irrigation sont sans doute les deux principaux facteurs qui relient la présence de variétés traditionnelles et commerciales. Dans le périmètre qui va du haut plateau près de la ville de Mexico (2400 $\mathrm{m}$ d'altitude) jusqu'aux altitudes intermédiaires dans l'État de Morelos (1200 m d'altitude) Perales et al. (2003a) rapportent que sur le haut plateau les variétés traditionnelles représentent $100 \%$ des lots de semences, tandis qu'à des altitudes moindres, les hybrides et les produits de leurs générations 
avancées sont très courants ( 34 et $37 \%$, respectivement). Ce fait est significatif, parce que la communauté près de la ville de Mexico a une meilleure infrastructure et un accès aux marchés, une meilleure éducation et quatre instituts de recherches proches avec plus de 50 ans de travaux d'amélioration du maïs. En outre, la production des variétés traditionnelles sur le haut plateau est principalement destinée au marché (66 \% de la production) et là où les hybrides sont présents, on les destine de préférence à la consommation (>70\%). Une situation similaire se présente au Chiapas (Brush \& Perales 2007) où dans des atmosphères semi-chaudes et tempérées (>1400 $\mathrm{m}$ asl) les variétés traditionnelles dominent complètement, avec une présence très limitée des variétés commerciales de générations avancées $(<1 \%)$ Au contraire, dans les climats chauds ( $<1400 \mathrm{~m}$ d'altitude) du Chiapas, les variétés commerciales sont assez communes $(40,8 \%)$ et un peu plus fréquentes chez les agriculteurs métis $(37,6 \%)$ que chez les agriculteurs indigènes (17,4 \%).

Arslan et Taylor (2009) ont utilisé un modèle économétrique pour un échantillon au niveau national et ont trouvé que l'irrigation était associée positivement comme facteur pour expliquer l'usage de variétés traditionnelles. La majeure partie du semis avec du maïs irrigué se trouve dans le nord du pays et les observations sommaires montrent que les variétés commerciales y sont dominantes. Cependant, dans certaines régions avec un sol de bonne qualité et une pluviosité suffisante, on utilise des semences commerciales. Arslan et Taylor ont aussi trouvé que l'aire ainsi cultivée était associée négativement avec la présence de variétés traditionnelles, c'est-à-dire qu'on la rencontre plus souvent chez de petits producteurs. La présence de variétés commerciales était aussi associée positivement à l'usage de machines et négativement au relief en pente, à la sécheresse et à l'altitude. Il est également évident que le bienêtre économique affecte l'utilisation de variétés traditionnelles: les foyers les moins pauvres font une plus grande utilisation des variétés commerciales (Bellon et al. 2006). Mais tous les groupes ayant un bien-être économique, étudiés dans l'Oaxaca comme au Chiapas, ont utilisé les deux types de variétés et leur utilisation dépendait plus des sortes de variétés, commerciales ou traditionnelles, principalement utilisées dans ce contexte. C'est-à-dire que dans l'Oaxaca on préférait les variétés traditionnelles et au Chiapas les commerciales, indépendamment du niveau de bien-être économique du groupe étudié. Il faut également noter qu'il n'y a pas d'association entre l'utilisation de fertilisants et d'herbicides et le type de variétés. Même si au Chiapas presque tous ( $95 \%)$ ceux qui sèment des variétés commerciales utilisent des fertilisants et des herbicides, plus de $70 \%$ de ceux qui sèment des variétés traditionnelles utilisent aussi ces produits (Perales, non publié). En outre, on a trouvé que l'adoption partielle de variétés commerciales était courante (Bellon 1991, Bellon \& Brush 1994), beaucoup d 'agriculteurs adoptent les semences commerciales sans écarter leurs variétés traditionnelles. Cela vient des caractéristiques contrastées entre les variétés principales, par exemple, les hybrides sont connus pour être plus résistants à la verse et mieux se vendre, et les variétés traditionnelles pour mieux résister au pourrissement des épis et aux insectes pendant le stockage. (Bellon et al. 2006).

Bien qu'on observe une grande variation morphologique entre les races, on a trouvé que la différence génétique entre celles-ci est relativement limitée comparée à la variation trouvée à l'intérieur de chaque race. Vigouroux et al. (2008) ont analysé 310 races et ont trouvé qu'à l'intérieur de ces races la variation était plus importante (24\%) que celle entre races $(7,6 \%)$. Dans cette étude ils ont aussi découvert que la différence 
génétique était davantage corrélée avec la distance géographique $(\mathrm{r}=0.41)$ et qu'avec 1 'altitude $(0,044)$ et le nombre des races $(0,065)$. Pressoir et Berthaud $(2004 a, b)$ ont étudié plusieurs communautés à Oaxaca et ont trouvé un niveau très bas d'isolement par la distance. Selon eux, la différenciation entre communautés est moindre qu'à 1 'intérieur de ces communautés: apparemment l'échange de semences est suffisamment vaste pour limiter la différenciation entre les communautés. Cependant, ils ont trouvé un nombre d'homozygotes plus grand qu'escompté par rapport à 1 'équilibre proposé de Hardet-Weinberg et celui-ci était en rapport avec l'amplitude de la période de floraison et l'intervalle entre l'apparition des fleurs mâle et des fleurs femelle. Reif et al. (2006) ont montré également que s'il y a des allèles singuliers dans les races de maïs, la majeure partie des gènes sont partagés entre races.

Il est remarquable que l'on rencontre ce modèle d'une plus grande diversité à 1 'intérieur des races (ou des communautés), bien que la quantité la plus grande d 'échanges de graines se produise à l'intérieur des communautés (Badstue et al. 2006, 2007, Brush \& Perales 2007). Baduste et al. $(2006,2007)$ ont étudié six communautés d 'Oaxaca et ont trouvé que $95 \%$ des semences avaient leur origine dans la communauté et étaient acquises grâce à des membres de la famille ou grâce à des connaissances. En une année, entre $23 \%$ et $39 \%$ des foyers ont fourni, ou se sont procurés des semences, même si dans toutes les communautés la présence de variétés traditionnelles était dominante ; dans cinq des six communautés la présence de semence commerciale était nulle ou inférieure à $4 \%$, dans une communauté elle arrivait à $14 \%$. Il est important que le principal motif signalé pour l'acquisition de semences soit l'expérimentation $(32,5 \%)$, pour essayer d'autres variétés, et non par manque de semence (18,2\%). Brush et Perales (2007) ont aussi trouvé que la communauté était l'origine principale des semences pour les foyers et que, dans les zones chaudes, il y avait une plus grande quantité de semences qui venaient de l'extérieur de la communauté (36\%) que dans les zones froides (5\%).

On s'attend généralement à ce que les variétés améliorées soient supérieures en termes de rendement aux variétés traditionnelles (Frankel 1970, Brush 1989). Dans le cas du maïs au Mexique cette généralisation est inappropriée surtout dans les zones semichaudes et tempérées. Perales (2016) a présenté les résultats d'un ensemble d 'expérimentations dans lesquelles les variétés traditionnelles de maïs ont un rendement égal ou supérieur à celui des variétés améliorées dans des conditions marginales ou dans les zones des hauts plateaux du centre du Mexique. Il est possible que les variétés traditionnelles se maintiennent parce qu'il y a des zones où elles sont meilleures, c'est-à-dire qu'elles sont compétitives du point de vue agronomique et/ou économique vis-à-vis des variétés améliorées (Perales et al. 1998). En général, on $\mathrm{s}$ 'attend à une forte adaptation locale des variétés de maïs, cependant, Orozco et al. (2014) ont montré que ce n'était pas le cas dans les communautés de Chatines et de Mixtèques de l'Oaxaca. Ils ont suggéré que des facteurs sociaux comme l'échange de semences et l'appartenance ethnique étaient plus importants que l'adaptation locale.

19 En plus des facteurs environnementaux et de productivité, la diversité observée dépend aussi de facteurs économiques et culturels (Leclerc \& d'Eeckenbrugge 2012). Il y a plus de 60 ans qu'Anderson (1947) a signalé que le "maïs est un miroir qui reflète les personnes qui le cultivent». Perales et al. (2005), ont comparé deux groupes ethnolinguistiques du Chiapas et ils ont montré que les différences dans les variétés de maïs ne pouvaient pas s'expliquer entièrement par l'adaptation à l'environnement, 
bien qu'ils aient trouvé un flux de gènes, et ils n'ont pas trouvé de différences dans la classification et le maniement des maïs par les paysans. Il se peut que les différences viennent des systèmes de semences et de la préférence à acquérir des semences à 1 'intérieur des communautés. Dans une autre étude Brush et Perales (2007) ont montré que les maïs préférés par la population indigène et métisse au Chiapas étaient similaires pour les environnements étudiés. Cependant, ils ont trouvé que les métisses avaient une légère préférence pour les variétés de la race Tuxpeño et les indigènes pour les variétés des espèces Olotón et Comiteco. En outre, les indigènes gardaient plus longtemps leurs semences et avaient une préférence plus grande pour les maïs colorés (jaunes, bleus et rouges.)

De ce fait on peut dire que la qualité des maïs est aussi un facteur qui intervient dans la diversité de ceux-ci, bien que nous n'en n'ayons guère qu'une information anecdotique. Au Mexique il y a des pratiques culinaires qui demandent des types spéciaux de maïs pour leur préparation. C'est le cas par exemple pour le pozole, cette soupe à base de grains de maïs que l'on fait bouillir jusqu'à ce qu'ils éclatent et pour laquelle il faut du maïs Ancho, Cacahuacintle ou Tabloncillo parce qu'ils ont des grains de grande taille qui éclatent bien. Mais ils coûtent jusqu'à deux ou trois fois plus cher que les maïs ordinaires. D'autres préparations spéciales peuvent demander aussi des maïs particuliers, par exemple l'espèce Bolita pour les clayudas (une sorte de grande tortilla (galette) sèche) ou du Zapalote chico pour les totopos (une tortilla grillée). Les marchés de maïs spéciaux sont en augmentation, par exemple, près de la ville de Mexico, la demande d'aliments pour les touristes a augmenté l'offre de plats préparés avec du maïs bleu (Perales et al. 2003b, Keleman \& Hellin 2009). Cependant on utilise principalement le maïs au Mexique pour faire les tortillas et on considère que presque toutes les variétés sont bonnes pour cela. Cela implique que, bien que la qualité compte, la plupart des maïs ne peuvent se vendre que comme maïs ordinaire au prix courant. Cependant, dans beaucoup de marchés ruraux les variétés traditionnelles ont un prix légèrement supérieur à celui des variétés commerciales, mais ce n'est pas le cas sur les marchés de masse, et l'industrie de la farine de maïs rejette les variétés traditionnelles à cause de la variabilité des grains, de taille plus grande que ceux des hybrides.

21 Un autre aspect important est que les variétés traditionnelles ne sont pas stables. On a écrit que certains agriculteurs cherchent à croiser des variétés traditionnelles avec des variétés commerciales (Perales et al. 2003b) et ce n'est pas rare (Bellon et al. 2006). On peut trouver le même processus entre des variétés traditionnelles. Perales et al. (2003b) ont décrit que dans un environnement semi-chaud de Morelos les agriculteurs ne reconnaissaient pas avoir hérité de leurs aïeux le maïs appelé Ancho-Pozolero. Ils reconnaissaient avoir hérité des maïs connus comme l'Ancho et ont dit qu'il y avait eu une introduction d'un maïs appelé Pozolero. Le Pozolero a été à l'origine du marché pour le pozole, qui permet un prix plus élevé que celui du grain de l'Ancho. Après 1 'introduction du Pozolero ils ont découvert qu'en sélectionnant leur maïs Ancho pour la taille de son grain, on pouvait le faire passer comme Pozolero et obtenir également un prix plus élevé. Ils n'étaient pas complètement d'accord sur le fait qu'ils aient seulement sélectionné leurs maïs Anchos ou qu'ils aient créé un type nouveau à partir du croisement de l'Ancho avec le Pozolero, mais il y avait consensus sur le fait que ce $\mathrm{n}$ 'était pas le même type de maïs que celui qu'ils avaient hérité de leurs parents. base de données de plus de 18000 entrées référencées géographiquement et classées 
par espèces. L'analyse de ces données montre que la diversité la plus grande, mesurée par la richesse en races, se trouve à l'ouest et dans le centre du pays; l'est est typiquement peu diversifié (Perales \& Golicher 2014). Mais il est intéressant de noter que sur les 59 races décrites, 47 seulement ont un échantillon suffisant pour être pris en compte dans l'analyse, cinq races sont très communes depuis les années 1940 alors que la moitié est rare ou même très rare, ce que Wellhausen et al. (1951) ont signalé il y a plus de 50 ans. L'abondance des espèces a été bien établie (Rho de Spearman : entre 0,63 et 0,72 ) sur les trois périodes de temps pendant lesquelles on a analysé les données (1943-1954, 1968-1979 et 1997-2009). De plus, toutes les espèces présentes dans les années 1950 ont été trouvées dans les récoltes récentes (1997-2009) et on n'a trouvé aucune diminution dans cette richesse au niveau national. À partir de cette analyse Perales et Golicher (2014) ont proposé 11 régions biogéographiques basées sur la composition raciale et six d'entre elles renferment $90 \%$ de toutes les collectes pour 38 des 47 espèces.

\section{L'amélioration évolutive et la sécurité alimentaire, une interprétation de la persistance du maïs natif au Mexique}

Deux faits sont remarquables à propos du maïs au Mexique au début du xxI siècle : la majeure partie de la semence appartient à des variétés traditionnelles et le nombre de foyers qui sèment du maïs n'a pas diminué malgré l'Accord de libre-échange nordaméricain, (en vigueur depuis 1994) et la diminution conséquente du prix du maïs. Il est possible qu'il faille interpréter ces deux faits ensemble.

Pourquoi l'abandon de la culture du maïs, attendu par certains après l'ALENA, ne s'estil pas produit? Quand l'ALENA a été mis en place, plusieurs auteurs ont supposé que 1 'on sèmerait moins de maïs au niveau national, on croyait en particulier que cette diminution affecterait ceux qui vendaient leur production plutôt que ceux qui produisaient seulement pour assurer leur subsistance et on s'attendait à une diminution de l'usage des variétés traditionnelles (Calva 1995, Janvry et al. 1995, Yúnez \& Barceinas 2001, Nadal et Wise 2004). En fait, le nombre des agriculteurs semant du maïs a augmenté légèrement entre le Censo Agrícola, Ganadero y Forestal, Recensement Agricole, de l'Elevage et Forestier de 1991 (2,75 millions) et celui de 2007 (2,83 millions). Les superficies ensemencées ont été relativement stables (7,7 Mha et 8,1 Mha) et la production a augmenté (14,3 Mt et 23,5 Mt), indiquant qu'il ne s'était pas produit un déclin dramatique de la production de maïs (Eakin et al. 2014). Cela a été possible parce que la majeure partie des agriculteurs, en particulier ceux qui sèment des variétés traditionnelles et ne vendent pas leur maïs, ne participent que partiellement aux marchés et offrent des prix "au noir » à l'intérieur de leurs unités de production (Arslan \& Taylor 2014). Il s'est produit cependant un changement important de la production de maïs dans les zones irriguées au nord du pays (Sweeney et al. 2013). Ces zones ont peu changé et, donc, bien qu'il se produise une diminution du nombre de lots de semences par agriculteur (Deter et al. 2014), il est possible qu'elle n'ait pas encore affecté la richesse des espèces (Perales \& Golicher 2014) et ces variétés sont toujours présentes et communes. Autrement dit, les variétés traditionnelles ont peu souffert de l'ALENA. 
Pourquoi une telle quantité de semences de variétés traditionnelles est-elle encore en usage ou, inversement, pourquoi les variétés commerciales ne sont-elles pas parvenues à s'imposer au Mexique? Les variétés commerciales ont été largement promues au Mexique depuis la décennie 60 , que ce soit à travers les paquets technologiques de la révolution verte ou avec l'obligation de les semer quand on donnait des crédits pour le faire. Cependant l'adoption des variétés commerciales s'est limitée aux zones chaudes parce que dans les zones tempérées leur adoption n'a pas dépassé en rendement celui des variétés traditionnelles (Perales et al. 2003a). Après la décennie 90 les variétés commerciales ont connu très peu d'expansion dans de nouvelles régions. Les raisons en sont variées :

- des conditions environnementales marginales comme des terres très en pente, ou avec des sols dégradés, pierreux, des précipitations rares et/ou un manque d'irrigation, - des facteurs sociaux et économiques, comme le manque d'investissement dans 1 'agriculture de subsistance, l'absence de conseil technique, le manque d'infrastructure et les défaillances du marché,

- des facteurs culturels, comme la qualité requise pour la préparation des aliments, la préférence pour les maïs locaux, ou le fonctionnement fondamentalement communautaire des systèmes d'échanges de semences.

Cependant on a proposé aussi une explication qui relève l'absence de sélectionneurs : selon Frankel et al. (1995: 84) « il se pourrait bien que (...) ce ne soit pas la supériorité des variétés traditionnelles qui soit la cause de leur maintien, mais l'absence de sélectionneurs locaux aptes à produire des variétés améliorées qui conviennent à 1 'agroécosystème ». Autrement dit, dès le début du travail théorique pour la conservation de la diversité génétique agricole, on a supposé que les agriculteurs ne garderaient pas leurs variétés traditionnelles face aux variétés commerciales "supérieures " (Frankel 1970), sinon les agriculteurs auraient à payer le coût de la conservation in situ ou à recevoir des aides (Frankel et al. 1995). C'est-à-dire qu'avec plus de sélectionneurs, plus de moyens et plus de temps, il n'y aurait plus de conservation in situ. Cependant, il se pourrait qu'il n'y ait jamais assez de sélectionneurs pour tous les environnements? Il se pourrait que la complexité environnementale et sociale soit telle que l'amélioration de plantes institutionnelles ne soit jamais le seul processus?

C. Suneson (1956), Bal et al. (1959) ont présenté il y a plus de 50 ans les résultats d'une expérimentation intéressante dans ce contexte. Suneson avait utilisé des mélanges issus du croisement de populations d'orge effectué par H.V. Harlan et il les a laissé évoluer par sélection naturelle pendant 29 générations. Quand on a comparé ces mélanges avec les variétés habituelles on s'est aperçu qu'elles étaient raisonnablement compétitives en ce qui concerne le rendement et la résistance aux maladies, parfois même légèrement supérieures aux cultures commerciales. Il faut cependant nuancer cette généralisation, selon Hockett et al. 1983, Soliman et Allard 1991. Suneson a donné à cette méthode le nom d' amélioration évolutive qui consiste en « la sélection naturelle concertée en temps et diversité dans la population » (Suneson \& Wiebe 1962, Suneson 1969) et ces dernières années elle a été remise en pratique pour l'agriculture biologique et l'agriculture à faible apport d'intrants (Voir par exemple: Murphet et al. 2004, Phillips and Wolfe 2005, Ceccarelli 2014).

28 Il est nécessaire de faire d'autres recherches sur la compétitivité et l'efficacité de 1 'amélioration évolutive pour différentes cultures dans différentes conditions, il est 
cependant possible de dire que ce mécanisme fonctionne dans le cas du maïs au Mexique. Le maïs présente au Mexique une grande variété génétique et phénotypique et ce sont deux millions d'agriculteurs qui sèment des variétés traditionnelles et gardent leur semence. Dans cette grande "loterie» des croisements et phénotypes (Bonneuil 2006) il y assurément certaines combinaisons réussies et les agriculteurs les distinguent et les sélectionnent. Il se peut que le processus soit lent mais, à la fin, il n 'est pas si éloigné de l'amélioration génétique classique pour laquelle, malgré la théorie et la capacité de prédiction des combinaisons susceptibles du plus grand succès, on ne peut pas éviter de faire beaucoup de croisements pour sélectionner les meilleurs. Il $n$ 'est pas encore possible d'affirmer nettement que l'amélioration évolutive soit le mécanisme qui a permis la compétitivité des variétés traditionnelles de maïs au Mexique, mais c'est un fait à considérer sérieusement. Si cela a été le cas, nous devons revoir la stratégie en vue de l'amélioration à long terme du maïs au Mexique pour que les agriculteurs et leurs variétés cessent d'être considérés comme du folklore et deviennent la partie irremplaçable du futur de l'évolution du maïs au Mexique.

En réunissant ces deux idées, il est possible d'expliquer la permanence de la conservation in situ du maïs au Mexique par un scenario d'amélioration évolutive dans le cadre $\mathrm{du}$ besoin de sécurité alimentaire des foyers. Même si les variétés traditionnelles n'ont pas été « créées » en tant que composés génétiques larges, elles le sont de fait. Elles se sont maintenues au Mexique en évoluant au cours des siècles et se sont renouvelées constamment avec les échanges de semences et les flux de pollen. Ces variétés continuent à changer et évoluent principalement par sélection naturelle et selon différents degrés de sélection artificielle. Tous ces efforts n'ont pas besoin de réussir. Cependant les populations qui se comportent bien sont sûrement distribuées dans les communautés. Le processus n'a pas besoin d'être très efficace ou rapide, mais il peut être adéquat pour ces agriculteurs et aussi bon sinon meilleur que les alternatives disponibles, qui, comme cela arrive souvent, n'ont pas été développées spécifiquement pour leur environnement. En outre comme ils envisagent leur production pour leur consommation ces agriculteurs restent un peu à l'écart du marché, ce qui leur permet de ne pas rechercher le maximum de rendement ou de revenus. Ces agriculteurs cherchent à avoir une production adaptée pour assurer la sécurité alimentaire de leurs foyers au moindre coût possible et non pour un maximum de rendement ou de revenus. S'ils étaient des agriculteurs conventionnels de type entrepreneurial, ils auraient sûrement abandonné la production de maïs. Même si 1 'amélioration évolutive n'est pas aussi efficace que l'amélioration conventionnelle, dans ce scénario elle peut fonctionner de manière satisfaisante pour ces agriculteurs et leurs familles et ils continueront de l'appliquer tant que ce sera le cas.

Après des millénaires où les agriculteurs et la sélection naturelle ont été les forces évolutives pour les espèces domestiquées, nous sommes aujourd'hui aveuglés par la croyance que les sélectionneurs, et en particulier les ingénieurs généticiens représentent la force principale et seule nécessaire pour faire évoluer les cultures. Imaginer que les scientifiques et techniciens soient les seuls pourvoyeurs de semence $n$ 'est pas fondé empiriquement et le fait que plus de la moitié du maïs semé au Mexique le soit avec des variétés traditionnelles est le signe que nous devons imaginer d'autres scénarios pour l'évolution du maïs au Mexique. Le défi n'est pas simple, cependant, à long terme, l'utilité et la compétitivité des variétés traditionnelles resteront 
primordiales pour leur conservation, et le processus aura certainement des potentialités pour une amélioration évolutive.

\section{Que devons-nous conserver?}

On a fait au Mexique plusieurs propositions pour la conservation de variétés traditionnelles de maïs. Le PROMAC, Programa de Maïs Criollo (Programme de Maïs Natif) a pris forme au sein de la Comisión Nacional de Áreas Naturales Protegidas ou CONANP (Commission Nationale d'Aires Naturelles Protégées) et s'est focalisé sur un système de subside. Bien qu'au début le nombre d'agriculteurs appuyés par le programme a été modeste par rapport à tous ceux qui cultivent ces variétés traditionnelles (approximativement $1 \%$ ), le nombre de bénéficiaires a été réduit rapidement entre 2009, début du programme, (25 826 agriculteurs) et 2013 (10 258 agriculteurs). Il y a un autre programme d'aides pour les groupements de "gardiens des races", soit quatre ou cinq agriculteurs qui ont la responsabilité de maintenir chaque race : en 2014 il y avait 214 gardiens pour 59 espèces. Un projet lié à Monsanto, le Proyecto Maestro de Maíces Mexicanos (Projet Maître en Maïs Mexicains), a inclus un financement pour les gardiens et a donné des aides à 186 agriculteurs en 2012, apparemment, il n'a pas eu de suite. En plus de ces actions institutionnelles, des organisations non gouvernementales ont mené plusieurs projets et actions, lesquels ont été limités dans leur portée ainsi que dans le temps. Que pouvons-nous attendre de cette approche et que voulons-nous ou de quoi avons-nous besoin pour la conservation du maïs mexicain ?

Le premier fait à considérer est l'échelle de la conservation de facto du maïs au Mexique : il s'agit de plus de deux millions d'agriculteurs et de plus de quatre millions $\mathrm{d}$ 'hectares. Si les objectifs formels de la conservation de ressources génétiques de maïs dans les champs des agriculteurs se limitent seulement à maintenir une représentation exagérément réduite de variétés traditionnelles, comme dans le cas des gardiens et des aides, quelle différence y aurait-il avec la conservation en banques de gènes ? Qu 'impliquerait-elle pour la conservation in situ du maïs? On pourrait objecter que cette conservation in situ aurait une valeur plus symbolique que pratique si sur des millions $\mathrm{d}$ 'agriculteurs seulement quelques centaines sont concernés. Il serait difficile de garantir la conservation des races avec si peu de cas et il serait plus improbable encore que ce petit nombre de cas suffisent à maintenir le processus évolutif. La probabilité de ce que dans cinq champs on trouve les réponses évolutives à de nouveaux fléaux ou maladies est très faible. La conservation in situ agricole exige d'être envisagée au niveau des communautés (de Boef et al. 2012) et non des individus. Un autre problème d'échelle est celui des aides. Le PROMAC est un exemple typique de la réduction continue d'un programme et de son application, sans parler de son maintien par des gouvernements différents. De tels efforts sont possibles dans le cadre d'un ensemble d'actions mais il est difficile qu'ils assurent une conservation in situ robuste et stable à long terme.

Un problème supplémentaire tient à la richesse et fréquence des espèces et variétés de maïs. On considère généralement que nous devons nous concentrer sur les zones de plus grande diversité et sur les races ou variétés les plus rares. S'il ne faut pas négliger ces aspects, il ne faut pas oublier non plus que les foyers ruraux mexicains dépendent des races et variétés communes pour leur subsistance. Au Mexique les espèces communes sont le fondement de la sécurité alimentaire, le fait qu'elles soient communes prouve qu'on les juge productives et qu'on peut compter sur elles année 
après année. Du point de vue de l'amélioration évolutive, au lieu de les écarter des programmes de conservation parce qu'elles ne risquent pas de disparaitre, il convient de faire des efforts pour qu'elles continuent à être compétitives et les mieux adaptées à leur environnement, comme elles le sont actuellement.

Du point de vue de l'amélioration évolutive, les races communes peuvent jouer un rôle particulier. L'importance de la continuité du processus évolutif a été mentionnée dans la littérature, même si on n'a pas spécifié qu'il en constitue l'essentiel. Par exemple, on a suggéré que la résistance aux insectes et maladies est importante, mais on peut également dire que l'aspect principal réside dans la participation des agriculteurs à la génération des variétés. La conservation du maïs dans les champs est le fait des agriculteurs qui sont les acteurs dans le processus de l'évolution des cultures, la présence de variétés traditionnelles est le témoignage de leur production de variétés pour leurs propres besoins. Les variétés qui évoluent entre les mains des agriculteurs ne garantiraient pas la permanence de ces mêmes variétés ni l'intégrité des formes historiques. Mais espérer que les agriculteurs cultivent à long terme sans qu'il y ait de changements dans leurs maïs, est peu crédible, et on a déjà mentionné que, selon Bennett, maintenir les gènes sans changement ne doit même pas représenter un objectif. La conservation dans les champs des agriculteurs doit servir d'abord au bienêtre des foyers qui cultivent le maïs, les gènes pour les généticiens peuvent en être le sous-produit, mais les gènes ne doivent être que cela, un sous-produit, et leur conservation ne peut pas être l'objectif principal. Il nous faut changer notre mentalité et passer de la volonté d'établir une banque de gènes à une perspective évolutive et centrée sur le bien-être des foyers des agriculteurs. Il nous faut imaginer un futur avec l'interaction complète des acteurs dans lequel les généticiens ne soient pas les seuls à traiter de l'évolution des cultures. Il n'y a aucune raison de penser que les agriculteurs cesseront d'être une force d'évolution des cultures agricoles. C'est peut-être dans le cadre de la conservation in situ que les agriculteurs montreront qu'ils continuent à fournir des solutions pour le futur.

Maxted N., Dulloo M.E. \& Ford-Lloetd B.V. (Ed.) 2016 - Enhancing crop genepool use: capturing wild relative and landrace diversity for crop improvement. CAB International, $469 \mathrm{p}$.

\section{BIBLIOGRAPHIE}

Aquino P., Carrión F., Calvo R. \& Flores D. 2001 - Selected maize statistics. In : Pingali P.L. (Ed.), CIMMETT 1999-2000 world maize facts and trends. Meeting world maize needs: technological opportunities and priorities for the public sector. CIMMETT, Mexico, D. F. : 45-57.

Aguirre J.A., Bellon M.R. \& Smale M. 2000 - A regional analysis of maize biological diversity in southeastern Guanajuato, Mexico. Economic Botany 54 : 60-72.

Anderson E. 1947 - Field studies of Guatemalan Maize. Annals of the Missouri Botanical Garden 34 : 433-467. 
Anderson E. \& Cutler H.C. 1942 - Races of Zea mays: I, Their recognition and classification. Annals of the Missouri Botanical Garden $29: 69-88$.

Appendini K. 2014 - Reconstructing the maize market in rural Mexico. Journal of Agrarian Change $14: 1-25$.

Aragón F., Taba S., Hernández J.M., Figueroa J.D., Serrano V. \& F.H. Castro F.G. 2006 - Catálogo de maíces criolles de Oaxaca. Etla, Oaxaca, INIFAP. (Libro Técnico ; 6).

Arslan A., Taylor J.E. 2009 - Farmer's subjective valuation of subsistence crops: the case of traditional maize in Mexico. American Journal of Agricultural Economics 91 : 956-972.

Badstue L.B., Bellon M.R., Berthaud J., Juárez X., Rosas I.M., et al. 2006 - Examining the role of collective action in an informal seed system: a case study from the central valleys of Oaxaca, Mexico. Human Ecology 34 : 249-273.

Badstue, L.B., Bellon, M.R., Berthaud, J., Ramírez, A., Flores, D., et al. 2007 - The dynamics of farmers' maize seed supply practices in the Central Valleys of Oaxaca, Mexico. World Development $35: 1579-1593$

Bal B.S., Suneson C.A. \& Ramage R.T. 1959 - Genetic shift during 30 generations of natural selection in barley. Agronomy Journal 51 : 555-557.

Bellon M.R. 1991 - The ethnoecology of maize variety management: a case study from Mexico. Human Ecology 19 : 389-418.

Bellon M.R. 2004 - Conceptualizing interventions to support on-farm genetic resource conservation. World Development $32: 159-172$.

Bellon M.R. \& Brush S.B. 1994 - Keepers of maize in Mexico. Economic Botany 48 : 196-209.

Bellon M.R. \& Risopoulos J. 2001 - Small-scale farmers expand the benefits of improved maize germplasm: a case study from Chiapas, Mexico. World Development 29 : 799-811.

Bellon M.R., Adato M., Becerril J. \& Mindek D. 2006 - Poor farmers' perceived benefits from different types of maize germplasm: the case of creolization in Lowland Tropical Mexico. World Development 34 : 113-129.

Bonneuil C. 2006 - Mendelism, plant breeding and experimental cultures: agriculture and the development of genetics in France. Journal of the History of Biology 39 :281-308.

Brush S.B. 1989 - Rethinking crop genetic resource conservation. Conservation Biology 3 : 19-29.

Brush S.B. 1995 - In situ conservation of landraces in centers of crop diversity. Crop Science 35 : 346-354.

Brush S.B. 2000 - The issues of in situ conservation of crop genetic resources. In : Brush S.B. (Ed.), Genes in the field, on farm conservation of crop diversity. IPGRI-IDRC-Lewis, Boca Raton, Florida, USA : 3-26.

Brush S.B. \& Perales H.R. 2007 - A maize landscape: Ethnicity and agro-biodiversity in Chiapas Mexico. Agriculture Ecosystems and Environment $121: 211-221$.

Calva J.L. 1995 - Razones et principios de una política integral incluetente de los campesinos. In : Moreno E. (Ed.), El sistema de poscosecha de granos en el nivel rural: problemática et propuestas.

Programa Universitario de Alimentos. No. 4. UNAM, Mexique : 79-140.

Ceccarelli S. 2014 - GM crops, organic agriculture and breeding for sustainability. Sustainability 6 : 4273-4286. 
De Boef S.W., Thijssen M.H., Shrestha P., Subedi A., Feetissa R., Gezu G., Canci A., da Fonseca M.A.J., Dias T., Swain S. \& Sthapit B.R. 2012 - Moving beyond the dilemma: practices that contribute to the on-farm management of agrobiodiversity. Journal of Sustainable Agriculture 36 : 788-809.

Deter G.A., López, A., Yúnez A. \& Taylor J.E. 2014 - Genetic erosion in maize's center of origin. Proceedings of the National Academy of Sciences of the United States of America 111 : 14094-14099.

Dyer G. A., López-Feldman A., Yúnez-Naude A. \& Taylor J.E. 2014 - Genetic erosion in maize's center of origin. Proceedings of the National Academy of Sciences, 111 (39) : 14094-14099.

Eakin H., Perales H., Appendini K. \& Sweeneet S. 2014 - Selling maize in Mexico: The persistence of peasant farming in an era of global markets. Development and Change 45 : 133-155.

Fernandez C., J. 2004 - The seed industry in U.S. agriculture. Agriculture Information Bulletin 786. U.S. Department of Agriculture, Economic Research System, 71 p.

Fox J. \& Haight L. 2010 - Subsidios por la desigualdad: las políticas públicas del maís del México a partir del libre comercio. Woodrow Wilson International Center for Scholars. Mexique, D.F.

Frankel O.H. 1970 - Genetic conservation in perspective. In : Frankel O.H. \& Bennett E. (Ed.) Genetic resources in plants: their exploration and conservation. IBP Handbook No. 11. Oxford, International Biological Program and Blackwell : 469-489.

Frankel O.H., Brown A.D.H. \& Burdon J.J. 1995 - The conservation of plant biodiversity. Cambridge, Cambridge University Press.

García J.A. \& Ramírez R. 2014 - El mercado de la semilla mejorada de maíz (Zea mays L.) en México: análisis del saldo comercial por entidad federativa. Revista Fitotecnia Mexicana 37 : 69-77.

Hewitt C. 1976 - Modernizing Mexican agriculture: Socioeconomic implications of technological change 1940-1970. Geneva, United Nations Institute for Research on Society and Development.

Hockett E.A., Eslick R.F., Qualset C.O., Dubbs A.L. \& Stewart V.R. 1983 - Effects of natural selection in advanced generations of barley Composite Cross II. Crop Science 23 : 752-756.

Janvry A. de, Chiriboga M., Colmenares H., Hintermeister A., Howe G., Irigoyen R., Monares A., Rello F., Sadoulet S., Secco J., van del Pluijm T. \& Varese S. 1995 - Reformas del sector agrícola y el campesinado en Mexico. San José, Costa Rica, Fondo Internacional de Desarollo Agrícola \& Instituto Interamericano de Cooperación por la Agricultura.

Jarvis D.I., Hodgkin T., Sthapit B.R., Fadda C. \& Lopez-Noriega I. 2011 - An heuristic framework for identifying multiple ways of supporting the conservation and use of traditional crop varieties within the agricultural production system. Critical Reviews in Plant Sciences, 30 (1-2) : 125-176.

Keleman A \& Hellin J. 2009 - Specialty maize varieties in Mexico: a case study in market-driven agro-biodiversity conservation. Journal of Latin American Geography 8 : 147-174.

Leclerc C. \& Coppens D’Eeckenbrugge G. 2012 - Social organization of crop genetic diversity. The G x E x S interaction model. Diversity $4: 1-32$ (doi:10.3390/d4010001).

Luna B.M., Hinojosa M.A., Aetala O.J., Castillo F. \& Mejía J.A. 2012 - Perspectivas de desarrollo de la industria semillera de maíz en México. Revista Fitotecnia Mexicana 35 : 1-7.

Marshall A. 2015 - Quality traits reach market. Nature Biotechnology 33 : 441.

Maxted N., Guarino L., Meter L. \& Chiwona E.A. 2002 - Towards a methodology for on-farm conservation of plant genetic resources. Genetic Resources and Crop Evolution 49 : 31-46. 
Murphet K., Lammer D., Letoin S., Carter B. \& Jones S.S. 2004 - Breeding for organic and lowinput farming systems: an evolutionary-participatory breeding method for inbred cereal grains. Renewable Agriculture and Food Systems 20 : 48-55.

Naciones Unidas 1993 - Convención de Diversidad Biológica. Rio de Janeiro.

Nadal A. 1999 - Issue Study 1. Maize in Mexico: some environmental implications of the North American Free Trade Agreement (NAFTA). In : Assessing environmental effects of the North American Free Trade Agreement (NAFTA): an analytic framework (Phase II) and issue studies. Montreal, Canada, Commission for Environmental Cooperation : 65-182.

Nadal A. 2000 - Mexican corn: genetic variability and trade liberalization. PROCIENTEC Documento de Trabajo1-06. Mexico, El Colegio de Mexico, $13 \mathrm{p}$.

Nadal A. \& Wise T.A. 2004 - The environmental costs of agricultural trade liberalization: Mexico-U.S. maize trade under NAFTA. Discussion Paper DP04. Working Group on Development and Environment in the Americas. Medford, Mass. USA, Tufts Universitet.

Orozco Q., Brush S.B., Grote M. \& Perales H. 2014 - Maize landrace distribution at local scale: the role of environmental adaptation. Economic Botany 68 : 383-396.

Perales H. 2016 - Landrace conservation of maize in Mexico: an evolutionary breeding interpretation. In : Maxted N., Dulloo M.E. \& Ford-Lloetd B.V. (Ed.), Enhancing crop genepool use, capturing wild relative and landrace diversity for crop improvement. Wallingford, $\mathrm{UK}, \mathrm{CAB}$ International : 271-281.

Perales H.R., Benz B.F. \& Brush S.B. 2005 - Maize diversity and ethnolinguistic diversity in Chiapas, Mexico. Proceedings of the National Academy of Sciences, USA 102 : 949-954.

Perales H., Brush S.B. \& Qualset C.O 1998 - Economic and agronomic competitiveness of maize landraces and in situ conservation in Mexico. In : Smale M. (Ed.), Farmers, gene banks and crop breeding: economic analyses of diversity in wheat, maize, and rice. Norwell, Massachusetts, CIMMETT and Kluwer Academic Publishers : 109-125.

Perales H., Brush S.B. \& Qualset C.O. 2003a - Landraces of Maize in Central Mexico: An Altitudinal Transect. Economic Botany 57 : 7-20.

Perales H., Brush S.B. \& Qualset C.O. 2003b - Dynamic management of maize landraces in Central Mexico. Economic Botany 57 : 21-34.

Perales H. \& Golicher D. 2014 - Mapping the diversity of maize races in Mexico. Plos ONE 9 (12) : e114657. (doi:10.1371/journal.pone.0114657).

Perales H. \& Hernández J.M. 2005 - Diversidad del maíz en Chiapas. In : González E., N. Ramírez M. \& L Ruiz M. (Ed.) Diversidad Biológica en Chiapas. Mexico, Plaza et Valdés : 419-440.

Phillips S.L. \& Wolfe M.S. 2005 - Evolutionary breeding for low input systems. Journal of Agricultural Science $143:$ 245-254.

Plucknett D. L., Smith N.J.H., Williams J. T. \& Murthi Anishettet N. 1987 - Gene banks and the world's food. Princeton, New Jersey, Princeton University Press.

Pressoir G. \& Berthaud J. 2004a - Patterns of population structure in maize landraces from the Central Valleys of Oaxaca in Mexico. Heredity 92 : 88-94.

Pressoir G. \& Berthaud J. 2004b - Population structure and strong divergent selection shape phenotypic diversification in maize landraces. Heredity $92:$ 95-101. 
PROMAC (Programa de Maíz Criollo). 2015 - Lineamientos y apoyos. En ligne : https:// www.conanp.gob.mx/maiz_criollo/pdf/maiz_2015/Lineamientos_PROMAC_2015.pdf.

Reif J.C., Warburton M.L., Xia X.C., Hoisington D.A., Crossa J., Taba S., Muminovic J., Bohn M., Frisch M. \& Melchinger A.E. 2006 - Grouping of accessions of Mexican races of maize revisited with SSR markers. Theoretical Applied Genetics $113: 177-185$.

SIAP (Servicio de Información Agroalimentaria et Pesquera) 2016 - Producción anual por cultivo, maíz. En ligne : www.siap.gob.mx/cierre-de-la-produccion-agricola-por-cultivo/ (consultado maeto 29, 2016).

Sanchez J.J., Goodman M.M \& Stuber C.W. 2000 - Isoenzimatic and morphological diversity in the races of maize of Mexico. Economic Botany 54 : 43-59.

Soliman K.M., \& Allard R.W. 1991 - Grain etield of Composite Cross populations of barleet: effects of natural selection. Crop Science 31, 705-708.

Suneson C.A. 1956 - An evolutionaret plant breeding method. Agronomet Journal 48, 188-191.

Suneson C.A. 1969 - Evolutionary plant breeding. Crop Science 9 : 119-121.

Suneson C.A. \& Wiebe G.A. 1962 - A « Paul Bunetan » plant breeding Enterprise with barley. Crop Science $2: 347-348$.

Sweeney S., Steigerwald D.G. Davenport F. \& Eakin H. 2013 - Mexican maize production: evolving organization and spatial structures since 1980. Applied Geography 39 : 78-92.

Vigouroux E.T., Glaubiktz J., Matsuoka E.T., Goodman M.M., Sanchez J. \& Doebleet J. 2008 Population structure and genetic diversity of New World maize races assessed bet DNA microsatellites. American Journal of Botany 95 : 1240-1253.

Yuñez-Naude A. \& Barceinas F. 2001 - Efectos de la desaparición de la Conasupo en el comercio y los precios de les cultivos básicos. Estudios Económicos 15 (2) : 189-227.

Wellhausen E.J., Roberts L.M., Hernández E. \& Mangelsdorf P.C. 1951 - Razas de maíz en México. Su origen, características y distribución. Oficina de Estudios Especiales, Secretaría de Agricultura et Ganadería. Mexique. D.F.

\section{RÉSUMÉS}

La conservation in situ des plantes cultivées, ou dans les champs des agriculteurs, a joué un rôle mineur dans la conservation des ressources génétiques et on lui a préféré les méthodes ex situ. Bien que l'on pense que la conservation à la ferme intéresse essentiellement les sélectionneurs généticiens, il faut considérer qu'elle a lieu car l'agriculture paysanne est encore liée aux variétés traditionnelles. Dans cet article le cas du maïs au Mexique est analysé selon cette perspective. La conservation de facto (sans intervention de la part des institutions), des variétés traditionnelles de maïs au Mexique est très étendue et dynamique, avec plus de deux millions d'agriculteurs qui sèment $d u$ maïs natif sur plus de la moitié des huit millions d'hectares ensemencés annuellement. Pour les petits agriculteurs, le maïs représente plus une sécurité alimentaire qu 'une source de revenus commerciaux, car il n'est pas actuellement la source principale de revenus pour la majeure partie d'entre eux. Plusieurs facteurs expliquent la présence de ces variétés traditionnelles de maïs dans le paysage tels que l'altitude, le sol de mauvaise qualité agricole, les systèmes d'échange de semences. Si l'on se réfère à la richesse des races, le centre et l'ouest du Mexique en offrent la plus grande diversité et cette richesse ne s'est pas réduite de façon significative dans les soixante dernières années. Les variétés traditionnelles de maïs ne 
sont pas statiques et certaines sont en train de changer pour répondre à des demandes spécifiques. On peut interpréter le cas du maïs au Mexique comme celui d'une amélioration évolutive liée à un objectif de sécurité alimentaire, là où les agriculteurs et la sélection naturelle d'une base génétique large produisent la semence indispensable pour des millions d'agriculteurs.

In situ conservation of crops, or conservation in farmers' fields, has been a minor component in the conservation of genetic resources; ex situ methods have been the preferred option. Although conservation in farmers' fields is thought to be aimed at conserving genes for geneticists, it happens because the well-being of many farmers' households depends on traditional varieties. A review of the case of corn in Mexico is presented. The de facto conservation, without institutional intervention, of traditional maize varieties in Mexico is extensive and dynamic with more than 2 million farmers planting native varieties, these are planted on more than half of the 8 million hectares planted annually. For small-scale farmers, maize represents food security, more than a business or monetary income, and maize is not currently the main source of income for most of these farmers. Several of the main factors that determine the presence of traditional maize varieties in the landscape are described, among which the altitude, the quality of the agricultural land, the quality of grains and seeds' flow. Based on maize races richness, central and western Mexico has the greatest diversity, and the richness has not significantly decreased in the last 60 years. Traditional maize varieties are not static and continue to change to meet specific demands. It is possible to interpret the case of maize in Mexico as evolutionary plant breeding within a food security objective, where farmers and natural selection of a broad genetic base continue to produce the seed that is essential for millions of farmers.

La conservación in situ de cultivos, o conservación en campos de agricultores, ha sido un componente menor en la conservación de recursos genéticos; los métodos ex situ han sido la opción preferida. Aunque se piensa que la conservación en campos de agricultores es destinada a conservar los genes para los genetistas, ésta sucede porque el bienestar de los hogares de muchos de los agricultores depende de variedades tradicionales. Se presenta una revisión del caso del maíz en México. La conservación de facto, sin intervención institucional, de las variedades tradicionales de maíz en México es extensa y dinámica con más que 2 millones de agricultores sembrando variedades nativas, éstas se siembran en más que la mitad de los 8 millones de hectáreas sembradas anualmente. Para los agricultores de pequeña escala el maíz representa seguridad alimentaria más que un negocio o ingreso monetario y actualmente el maíz no es el ingreso principal de la mayoría de estos agricultores. Se describen varios factores principales que determinan la presencia de las variedades tradicionales de maíz en el paisaje, entre los que destacan la altitud, la calidad de las tierras agrícolas, la calidad del grano y el intercambio de semillas. Con base en la riqueza de razas, el centro y oeste de México tiene la mayor diversidad y la riqueza no se ha reducido significativamente en los últimos 60 años. Las variedades tradicionales de maíz no son estáticas y siguen cambiando para responder a demandas específicas. Es posible interpretar el caso del maíz en México como mejoramiento evolutivo dentro de un objetivo de seguridad alimentaria, donde los agricultores y el mejoramiento evolutivo de una base genética amplia siguen produciendo la semilla indispensable para millones de agricultores. 
INDEX

Mots-clés : conservation in situ, variétés traditionnelles, sélection évolutive, sécurité

alimentaire

Palabras claves : conservación in situ, variedades tradicionales, mejoramiento evolutivo,

seguridad alimentaria

Keywords : in situ conservation, traditional varieties, evolutionary plant breeding, food security

\section{AUTEUR}

HUGO PERALES

El Colegio de la Frontera Sur, San Cristóbal, Chiapas, Mexique. 